\title{
Effect of everolimus on the immunomodulation of the human neutrophil inflammatory response and activation
}

\author{
Damien Vitiello $^{1,2,3}$, Paul-Eduard Neagoe ${ }^{1,2}$, Martin G Sirois $^{1,2}$ and Michel White $e^{1,3}$
}

The primary cause of mortality at 5 years following a cardiac transplantation is the development of atherosclerosis, termed coronary allograft vasculopathy (CAV). This pathology is characterized by diffused intimal hyperplasia and emanates from coronary arterial injuries caused by immune inflammatory cells. Neutrophils play an important role in this inflammatory process; however, their potential participation in the pathogenesis of CAV is poorly understood. Despite their essential contribution to the prevention of graft rejection, immunosuppressive drugs could have detrimental effects owing to their pro-inflammatory activities. Thus, we investigated the impact of different immunosuppressive drugs on the inflammatory response of neutrophils isolated from the blood of healthy volunteers. Under basal conditions, mammalian target of rapamycin (mTOR) inhibitors (sirolimus and everolimus) had the most potent anti-inflammatory effect, decreasing both IL-8 release $(\approx-80 \%)$ and vascular endothelial growth factor (VEGF) release $(\approx-65 \%)$ and preserving the release of the anti-inflammatory cytokine interleukin-1 receptor antagonist (IL-1RA). In TNF- $\alpha$-treated neutrophils, pre-incubation with everolimus provided the most potent effect, simultaneously reducing the release of both VEGF and IL-8 while doubling the release of IL-1RA. This latter effect of everolimus was maintained even when administered in combination with other immunosuppressive drugs. Sirolimus and everolimus decreased the tumor necrosis factor (TNF)- $\alpha$-induced adhesion of neutrophils to human endothelial cells and human extracellular matrix. This effect was largely dependent on the ability of these compounds to alter $\boldsymbol{\beta}_{2}$-integrin/CD18 activation. Our results suggest a potential mechanism for the beneficial effect of everolimus in the prevention of CAV in heart transplant recipients.

Cellular \& Molecular Immunology (2015) 12, 40-52; doi:10.1038/cmi.2014.24; published online 2 June 2014

Keywords: cytokines; human; immunology; immunosuppressive drugs; inflammation; neutrophils

\section{INTRODUCTION}

The principal cause of mortality at 5 years following cardiac transplantation (CTX) is the development of diffuse and accelerated cardiac atherosclerosis, termed coronary allograft vasculopathy (CAV). ${ }^{1}$ The development of CAV is multifactorial and is initially triggered by heterogeneous factors causing endothelial cell injury and a vascular inflammatory response. ${ }^{2}$ CAV is predominantly characterized by diffused intimal fibroelastic proliferation ${ }^{3}$ resulting from exaggerated vascular repair, ${ }^{4}$ which reduces the vascular coronary luminal diameter. Several immune cells are involved in this intimal proliferation, including monocytes/macrophages and $\mathrm{T}$ cells. Early infiltration of T cells after CTX interact with donor endothelial cells, triggering T-cell activation and the release of pro-inflammatory cytokines such as interleukin-2 (IL-2) and tumor-necrosis factor- $\alpha$ (TNF- $\alpha$ ), which increase the expression of intercellular adhesion molecule-1 (ICAM-1), vascular cell adhesion molecule- $1^{5}$ and E-selectin. ${ }^{6}$ Together, these events cause circulating leukocytes to adhere and transmigrate through the vessel wall. ${ }^{7}$ Additionally, activated monocytes/macrophages and lymphocytes release several growth factors, cytokines and chemoattractants. Upon their infiltration in the intima, monocytes/macrophages and lymphocytes induce the migration and proliferation of vascular smooth muscle cells into the intima, thus compromising the lumens of the coronary arteries. ${ }^{2,8,9}$ Together, these data suggest that activated immune cells and the related inflammatory response are involved in the intimal hyperplasia of coronary arteries resulting from endothelial injury and the development of CAV post-transplantation.

${ }^{1}$ Research Center, Montreal Heart Institute, Université de Montréal, Montreal, QC, Canada; ${ }^{2}$ Department of Pharmacology, Faculty of Medicine, Université de Montréal, Montreal, QC, Canada and ${ }^{3}$ Department of Medicine, Faculty of Medicine, Université de Montréal, Montreal, QC, Canada Correspondence: Dr M White, Université de Montréal, Research Center, Montreal Heart Institute, 5000 Belanger Street, Montreal H1T 1C8, Canada. E-mail: m_white@icm-mhi.com

Dr MG Sirois, Université de Montréal, Research Center, Montreal Heart Institute, 5000 Belanger Street, Montreal H1T 1C8, Canada. E-mail: martin.sirois@icm-mhi.org

Received: 21 January 2014; Revised: 20 February 2014; Accepted: 16 March 2014 
Similar to monocytes/macrophages and lymphocytes, neutrophils are immune cells that can participate in the development of CAV after CTX in heart transplant recipients. Neutrophils are the most abundant type of white blood cells in mammals and are known to play important roles in inflammation. ${ }^{10-12}$ Previous studies have demonstrated the potential deleterious role of neutrophils during myocardial reperfusion immediately after CTX in rodents, notably via neutrophil activated $\alpha_{\mathrm{M}} / \beta_{2}$-integrin complexes $(\mathrm{CD} 11 \mathrm{~b} / \mathrm{CD} 18) .^{13,14}$ Because neutrophils are able to release numerous mediators, including pro-inflammatory (e.g., IL-1 $\alpha / \beta,-6,-7,-8,-9,-16$ ), pro-inflammatory/angiogenic (e.g., tumor growth factor (TGF)- $\alpha$, vascular endothelial growth factor (VEGF) and anti-inflammatory mediators (e.g., IL-1 receptor antagonist (IL-1RA), $-4,-10$, TGF- $\beta$ ) ${ }^{10,15}$ under the inflammatory state usually observed in heart transplant recipients, ${ }^{16}$ they could also be involved in the development and/or the progression of CAV following CTX. Notably, previous studies have demonstrated the expression of chemokines and cytokines following solid organ transplantation in rodents ${ }^{17,18}$ and humans. ${ }^{19}$ In particular, the expression of IL- 8 has been observed in the cardiac allografts of mice at the early onset of $\mathrm{CAV},{ }^{17}$ and an increase in interferon-inducible $\mathrm{T}$ cell- $\alpha$ chemoattractant serum levels has been associated with the development of transplant coronary artery disease in heart transplant recipients. ${ }^{19}$ These data suggest that these early inflammatory events can allow the recruitment of neutrophils in the cardiac allograft, which can induce inflammation, thus favoring the development of CAV. In addition, another study reported that $\beta_{2}$-integrin-mediated neutrophil infiltration and accumulation in the cardiac allograft tissue was associated with acute rejection in mice. ${ }^{14}$ Together, these data suggest that neutrophils may be one of the immune cells that exacerbate the progression of the acute rejection of cardiac allografts. Finally, Raichlin et al. ${ }^{20}$ demonstrated that high plasma C-reactive protein levels have been associated with an increased risk of developing CAV in heart transplant recipients. This finding reinforces the concept that pro-inflammatory mediators can play an important role in the development of CAV in heart transplant recipients.

Inflammation is not the only factor involved in the pathogenesis of CAV. Immunosuppressive drugs (IDs), despite their essential role at preventing graft rejection after solid organ transplantation, are also considered a transplant-associated risk factor for CAV based on their pro-inflammatory activity. ${ }^{2}$ Badiwala et al. ${ }^{21}$ demonstrated that the calcineurin inhibitor cyclosporine A (CsA) induces injuries by increasing ICAM-1 expression on human coronary artery endothelial cells, thus also increasing neutrophil adhesion, which could ultimately increase local inflammation and favor the development of CAV. Among the different classes of IDs, the pro-drug mycophenolate mofetil and its active form (mycophenolate acid (MPA)) lead to the inhibition of inosine-5'-monophosphate dehydrogenase. This ID is efficient at preventing both the development of CAV at 1 year post-transplant by reducing the incidence of vessel shrinkage and the progression of intimal hyperplasia ${ }^{22}$ and by preventing episodes of acute rejection in heart transplant recipients. ${ }^{23}$ However, MPA has also been shown to cause neutropenia by inhibiting IL-17 expression in mice, ${ }^{24}$ which can be detrimental to patients because neutrophils are known to be crucial for host defense in sepsis. ${ }^{25}$ Finally, the third class of ID used in CTX is mTOR inhibitors, namely, sirolimus (SIR) and everolimus (EVE). SIR was shown to provide long-term benefits by mitigating CAV progression and reducing intimal hyperplasia in heart transplant recipients. ${ }^{26}$ In addition, the novel mTOR inhibitor EVE has been reported to partially prevent the development of $\mathrm{CAV},{ }^{27}$ to improve established CAV in heart transplant recipients ${ }^{28}$ and to be safe in combination with other IDs. ${ }^{29}$ However, these mTOR inhibitors have also been shown to have negative effects that could promote the development of CAV in heart transplant recipients. For instance, SIR treatment increased the risk of venous thromboembolism, ${ }^{30}$ and long-term treatment with EVE was associated with a significant increase in necrotic and calcified tissue in the human cardiac allograft. ${ }^{31}$ Thus, as IDs, these drugs can potentially have deleterious effects that are associated with the development of CAV in humans. Human neutrophils can also be associated with this condition owing to their ability to infiltrate the cardiac allograft and release numerous pro-inflammatory cytokines.

In the current study, we assessed the impact of different immunosuppressive drug regimens on the inflammatory response of neutrophils isolated from the venous blood of healthy volunteers to delineate potential ID regimens that can possibly be used in heart transplant recipients.

\section{MATERIALS AND METHODS}

\section{Study patients}

This study involved 58 healthy volunteers ( 34 men, 24 women; aged $24-65$ years; mean age: $43.6 \pm 1.5$ years) who were free from any medical condition or had not taken medication for at least 10 days prior to the experiments. The study was conducted in accordance with the Declaration of Helsinki and approved by the Montreal Heart Institute's ethical committee (Montreal, QC, Canada; ethics no. ICM \#01-406). All healthy volunteers provided written informed consent to the experimental protocol before participating in the study.

\section{Neutrophil isolation and purification}

Venous blood was obtained from healthy donors free from medication for at least 10 days prior to the experiments. Venous blood was obtained by drawing $100 \mathrm{ml}(4 \times 25 \mathrm{ml})$ of blood using a $21 \mathrm{G}$ needle into $30 \mathrm{ml}$ syringes prefilled with $5 \mathrm{ml}$ of Anticoagulant Citrate Dextrose Solution USP Formula A (Baxter Healthcare, Deerfield, IL, USA). The blood was then transferred into $4 \times 50 \mathrm{ml}$ tubes and spun for $15 \mathrm{~min}$ at $200 \mathrm{~g}$ at room temperature. Following centrifugation, the platelet rich plasma was removed from the top layer, and $20 \mathrm{ml}$ of a $4 \%$ Dextran solution $(138 \mathrm{mM} \mathrm{NaCl}, 5 \mathrm{mM} \mathrm{KCl}, 0.34 \mathrm{mM}$ $\mathrm{Na}_{2} \mathrm{HPO}_{4}, 0.4 \mathrm{mM} \mathrm{KH} \mathrm{PO}_{4}, 4.2 \mathrm{mM} \mathrm{NaHCO} 3,5.6 \mathrm{mM}$ Glucose, $10 \mathrm{mM}$ HEPES, $12.9 \mathrm{mM}$ sodium citrate and 250 mM Dextran; pH 7.4) was added per tube. The tubes were gently mixed, and the red blood cells were left to sediment for 
$45 \mathrm{~min}$ at room temperature. The upper layer containing the white blood cells was collected and gently deposited on a $12.5 \mathrm{ml}$ layer of Ficoll-Paque Plus (GE Healthcare, Baie d'Urfé, QC, Canada) in $50 \mathrm{ml}$ tubes before being spun for $28 \mathrm{~min}$ at $400 \mathrm{~g}$ at room temperature. Following this centrifugation, the monocytes and lymphocytes were separated from the neutrophils by Ficoll gradient. The remaining red blood cells and neutrophils were found in the pellet. To remove the red blood cells from the neutrophils, we used a water lysis procedure, in which we added $20 \mathrm{ml}$ of distilled water to the neutrophil and red blood cell pellet and mixed gently for $20 \mathrm{~s}$, followed by the quick addition of $20 \mathrm{ml}$ of Hank's balanced salt solution (HBSS) $2 \times$ solution while continuing mixing, for a final concentration of $1 \times$ HBSS ( $\mathrm{pH}$ 7.4). Neutrophils were then spun for $10 \mathrm{~min}$ at $200 \mathrm{~g}$ at room temperature. The pellet was then resuspended in RPMI 1640 medium (Lonza, Basel, Switzerland) supplemented with $25 \mathrm{mM}$ HEPES ( $\mathrm{N}$-2-hydroxyethylpiperazine- $N^{\prime}$-2-ethane sulfonic acid) and $1 \%$ penicillin/streptomycin. The contamination of the isolated neutrophil suspension with peripheral blood mononuclear cells was less than $0.5 \%$, as confirmed by Wright-Giemsa staining, and the viability was found to be greater than $98 \%$, as assessed by Trypan blue dye exclusion assay. ${ }^{32,33}$

\section{Neutrophil stimulation and treatment}

Purified neutrophils $\left(5 \times 10^{6} / \mathrm{ml}, 500 \mu \mathrm{l}\right)$ were incubated in RPMI-1640 solution (Gibco, Carlsbad, CA, USA) supplemented with 5\% fetal bovine serum (PAA Laboratories, Etobicoke, ON, Canada), 1\% penicillin/streptomycin/GlutaMAX (P/S) (Gibco) and $25 \mathrm{mM}$ HEPES (Sigma, Oakville, ON, Canada), hereafter termed RPMI (for complete RPMI-1640 solution). In the first series of experiments, neutrophils were stimulated for 2 and $24 \mathrm{~h}$ with control vehicle (phosphate-buffered saline (PBS)), N-Formyl-Met-Leu-Phe (fMLP; $\left.10^{-7} \mathrm{M}\right)$ (Sigma), bacterial lipopolysaccharide (LPS; Escherichia coli 0111:B4; $1 \mu \mathrm{g} / \mathrm{ml}$ ) (Sigma) or TNF- $\alpha(10 \mathrm{ng} / \mathrm{ml})$ (Peprotech, Rocky Hill, NJ, USA) at $37{ }^{\circ} \mathrm{C}$ and $5 \% \mathrm{CO}_{2}$. Following stimulation, neutrophils were centrifuged at $900 \mathrm{~g}$ for $7 \mathrm{~min}$, and the supernatants were stored at $-80{ }^{\circ} \mathrm{C}$ for future ELISA quantification of IL-1RA, IL-8 and VEGF (R\&D Systems, Minneapolis, MN, USA) according to the manufacturer's instructions. The aforementioned agonists (i.e., fMLP, LPS or TNF- $\alpha$ ) were used because of their capacity to promote IL-1RA, IL-8 and VEGF release by the neutrophils. ${ }^{33-36}$ In another set of experiments, neutrophils $\left(5 \times 10^{6} / \mathrm{ml} ; 500 \mu \mathrm{l}\right)$ were pre-treated with immunosuppressive drugs that are routinely used in heart transplant recipients, such as CsA, tacrolimus (TAC), MPA, SIR or EVE. Pre-treatment of neutrophils was performed using IDs alone or in combination at three different concentrations (i.e., CsA (10, $100,1000 \mathrm{ng} / \mathrm{ml})$, TAC $(1,10,100 \mathrm{ng} / \mathrm{ml})$, MPA $(20,200$, $2000 \mathrm{ng} / \mathrm{ml}), \operatorname{SIR}(1,10,50 \mathrm{ng} / \mathrm{ml})$ and $\operatorname{EVE}(1,10,100 \mathrm{ng} / \mathrm{ml}))$ for $30 \mathrm{~min}$ prior to agonist stimulation for 2 and $24 \mathrm{~h}$. To address the effect of various immunosuppressors on the release of cytokines by the neutrophils at early and late time periods, we elected to address the effect of the immunosuppressors on the release of VEGF and IL-1RA at $2 \mathrm{~h}$, and on the release of
IL-8 at 24 h post-stimulation. Following stimulation, neutrophils were centrifuged at $900 \mathrm{~g}$ for $7 \mathrm{~min}$, and supernatants were collected and stored at $-80^{\circ} \mathrm{C}$ for subsequent quantification of IL-1RA, IL-8 and VEGF by ELISA. The ID concentrations were selected based on the regimen provided to patients undergoing various organ transplants. ${ }^{37-39}$

\section{Neutrophil adhesion experiments}

Neutrophil adhesion to human umbilical vascular endothelial cells (HUVECs) or human extracellular matrix (hECM; BD Bioscience, Mississauga, ON, Canada) was measured under static conditions as described previously. ${ }^{40}$ HUVECs were seeded (40000 cells/well; 24-well plates) and cultured in endothelial basal medium 2 (Clonetics, San Diego, CA, USA) containing $10 \%$ fetal bovine serum (PAA Laboratories), endothelial growth medium 2 singlequot (Clonetics) and 2\% antibiotics (penicillin and streptomycin; Sigma, St Louis, MO, USA). HUVECs were used at passages 3-5. Three (3) days post-confluent HUVECs were rinsed with PBS $\left(37{ }^{\circ} \mathrm{C}\right)$. $\mathrm{HBSS}+\mathrm{CaCl}_{2}(5 \mathrm{mM})$ (termed HBSS $^{+}$) was added to HUVECs at $37^{\circ} \mathrm{C}$. For the adhesion experiments, neutrophils $\left(2 \times 10^{5}\right.$ cells in $\left.250 \mu \mathrm{HBSS}^{+}\right)$were pre-treated with or without immunosuppressive drugs ( $30 \mathrm{~min}$ ) alone or in combination, and when applicable, a blocking human $\beta_{2}$-integrin/CD18 antibody $(2 \mu \mathrm{g} / \mathrm{ml}$, Gln23-Asn700, Accession Number \#AAA59490; R\&D Systems) was added to neutrophils $15 \mathrm{~min}$ prior to the addition of neutrophils onto HUVECs and the addition of PBS or TNF- $\alpha(10 \mathrm{ng} / \mathrm{ml})$ for $7.5 \mathrm{~min}$. The wells were carefully rinsed with PBS to remove non-adherent neutrophils, and the adhered neutrophils were then fixed with a $1 \%$ paraformaldehyde/ $1 \%$ glutaraldehydePBS solution. Adhesion of the neutrophils to HUVEC monolayers was assessed using a color video digital camera adapted to a binocular microscope. For each well, four fields of view were randomly selected, and the neutrophils in each field of view were counted and recorded as the number of adhered neutrophils/ $\mathrm{mm}^{2}$. For the adhesion assay of neutrophils onto hECM, 24-well plates were coated with $250 \mu \mathrm{l} /$ well of hECM diluted in serumfree RPMI at a concentration of $20 \mu \mathrm{g} / \mathrm{ml}$. Briefly, the plates were incubated with the hECM dilution for $2 \mathrm{~h}$ at room temperature, washed twice with serum-free RPMI ( $250 \mu \mathrm{l} /$ wash $)$ and dried for a minimum of $2 \mathrm{~h}$. The neutrophils were then treated and added to hECM, as described for the HUVEC experiments.

\section{Statistical analysis}

All results were expressed as the mean \pm s.e.m. Statistical comparisons were made by a one-way analysis of variance, followed by a Bonferroni $t$-test using Statview 5.0 (SAS Institute Inc., Cary, NC, USA). Differences were considered significant at $P$ values $\leqslant 0.05$.

\section{RESULTS}

\section{Effect of pro-inflammatory agonists on the release of selected cytokines by neutrophils}

We assessed the capacity of selected pro-inflammatory agonists to induce the release of cytokines that are known to be expressed and released by human neutrophils. ${ }^{10,15}$ We assessed 
the release of VEGF (pro-inflammatory/pro-angiogenic mediator), IL-1RA (anti-inflammatory cytokine) and IL-8 (proinflammatory cytokine). Treatment with fMLP $\left(10^{-7} \mathrm{M}\right)$, LPS $(1 \mu \mathrm{g} / \mathrm{ml})$ and TNF- $\alpha(10 \mathrm{ng} / \mathrm{ml})$ induced the release of VEGF, IL-1RA and IL-8 by neutrophils $\left(5 \times 10^{6} / \mathrm{ml}, 500 \mu \mathrm{l}\right)$ (Figure 1 ). The quantification of VEGF and IL-1RA proteins was performed at $2 \mathrm{~h}$ post-stimulation, and the quantification of IL-8 protein occurred at $24 \mathrm{~h}^{15,41}$ All pro-inflammatory agonists significantly increased the release of VEGF. The most potent effect was evoked by LPS, corresponding to a $403 \%$ increase compared with PBS-treated neutrophils. For the release of IL-1RA, there was a significant $110 \%$ and $151 \%$ increase induced by LPS and TNF- $\alpha$, respectively, whereas fMLP had no significant effect. All pro-inflammatory agonists increased the release of IL- 8 by the neutrophils. LPS induced a 65 -fold $(6400 \%)$ increase, whereas fMLP and TNF- $\alpha$ produced more modest increases in IL-8 release (213\% and 595\%, respectively) (Figure 1).

\section{Effect of IDs on cytokine release by neutrophils under basal conditions}

We investigated the capacity of CsA $(10,100,1000 \mathrm{ng} / \mathrm{ml})$, TAC $(1,10,100 \mathrm{ng} / \mathrm{ml}), \operatorname{MPA}(20,200,2000 \mathrm{ng} / \mathrm{ml})$, SIR $(1,10$, $50 \mathrm{ng} / \mathrm{ml})$ and $\operatorname{EVE}(1,10,100 \mathrm{ng} / \mathrm{ml})$, alone or in combination, to alter the release of VEGF, IL-1RA and IL- 8 by neutrophils under basal (PBS) and stimulated (fMLP, LPS and TNF- $\alpha$ ) conditions. To facilitate data interpretation, the basal values of VEGF, IL-1RA and IL-8 release under the PBS-treated condition were set to 1 , and the increases mediated by fMLP, LPS and TNF- $\alpha$ were expressed as the fold increase (Figures 2-4).

Under the basal (PBS) condition, pre-treatment of neutrophils with IDs, individually or in combination, significantly decreased the release of VEGF (Figure 2a), with the highest decreases induced by mTOR inhibitors (SIR and EVE; 65\% reduction). Pre-treatment of neutrophils with IDs, alone or in combination, had no or very slight effects on IL-1RA release (Figure 3a). Most pre-treatments with IDs, alone or in combination, significantly decreased IL-8 release (Figure 4a). However, at their highest concentrations, SIR or TAC had no effect or increased IL-8 release, respectively. The greatest inhibition of basal IL-8 release was observed when neutrophils were pre-treated with EVE alone (-90\%) or in combination with another ID (Figure 4a).

\section{Effect of IDs on VEGF release by neutrophils under pro- inflammatory conditions}

Pre-treatment with CsA did not have a significant effect on VEGF release under all pro-inflammatory stimulations (Figure $2 \mathrm{~b}-\mathrm{d}$ ). Pre-treatment with TAC and MPA had no significant effect on fMLP-induced VEGF release, but it did induce a significant decrease in VEGF release under LPS and TNF- $\alpha$ stimulations, where the most efficient inhibitory effect was obtained at the lowest concentrations (Figure $2 c-d$ ). Pretreatment with SIR and EVE, alone or in combination with CsA or TAC, significantly decreased the release of VEGF by neutrophils following all pro-inflammatory stimulations (Figure $2 \mathrm{~b}-$ d). The effect of these two IDs was nearly equivalent, reducing the VEGF release induced by fMLP, LPS and TNF- $\alpha$ by $88 \%-$ $100 \%$ compared with the PBS-treated cells (Figure 2b-d). Notably, when SIR was combined with MPA, we observed a complete or partial loss of the capacity of SIR to prevent VEGF release, whereas the capacity of EVE was maintained upon its combination with MPA (Figure 2b-d).

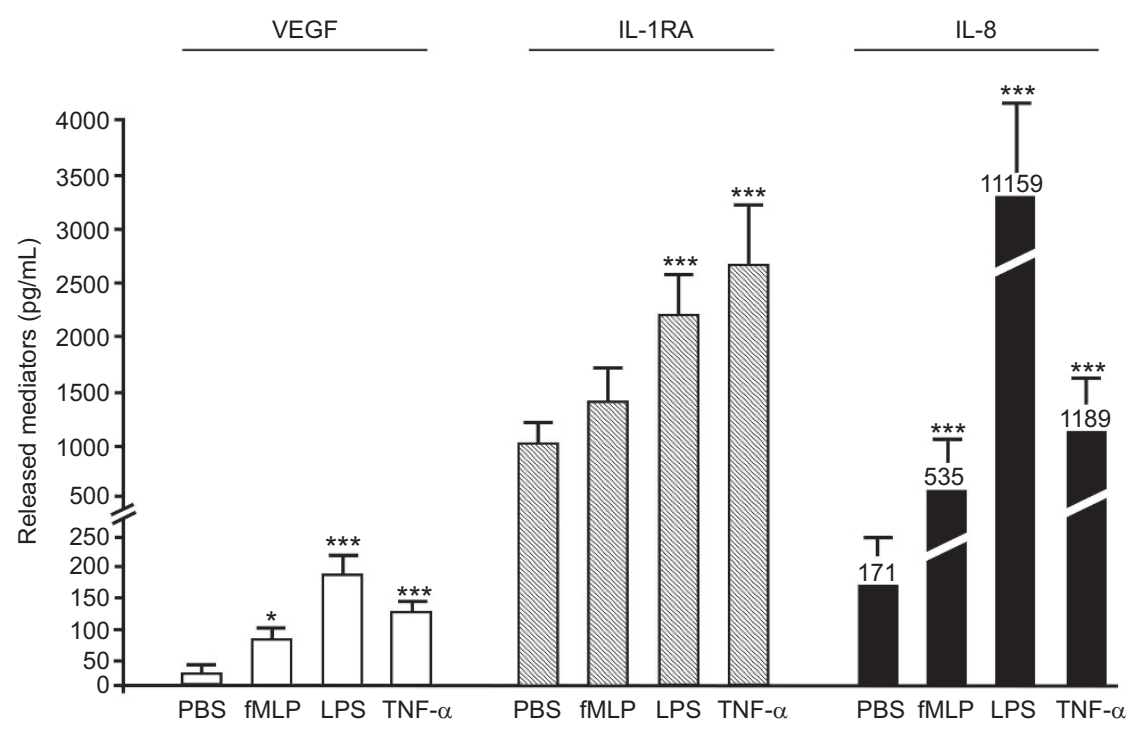

Figure 1 Effect of pro-inflammatory agonists on the release of inflammatory cytokines by neutrophils. Neutrophils were incubated for 2 and $24 \mathrm{~h}$ in presence of PBS (vehicle), fMLP $\left(10^{-7} \mathrm{M}\right), \mathrm{LPS}(1 \mu \mathrm{g} / \mathrm{ml})$ or TNF- $\alpha(10 \mathrm{ng} / \mathrm{ml})$ for the subsequent quantification of VEGF, IL-1RA $(2 \mathrm{~h})$ and IL- 8 (24 h) release by ELISA. Data are represented as the mean \pm s.e.m. of at least eight independent experiments. $* P<0.05$ and $* * * P<0.001$ compared with PBS-treated neutrophils. fMLP, Formyl-Met-Leu-Phe; IL-1RA, interleukin-1 receptor antagonist; LPS, lipopolysaccharide; PBS, phosphate-buffered saline; TNF- $\alpha$, tumor-necrosis factor- $\alpha$; VEGF, vascular endothelial growth factor. 

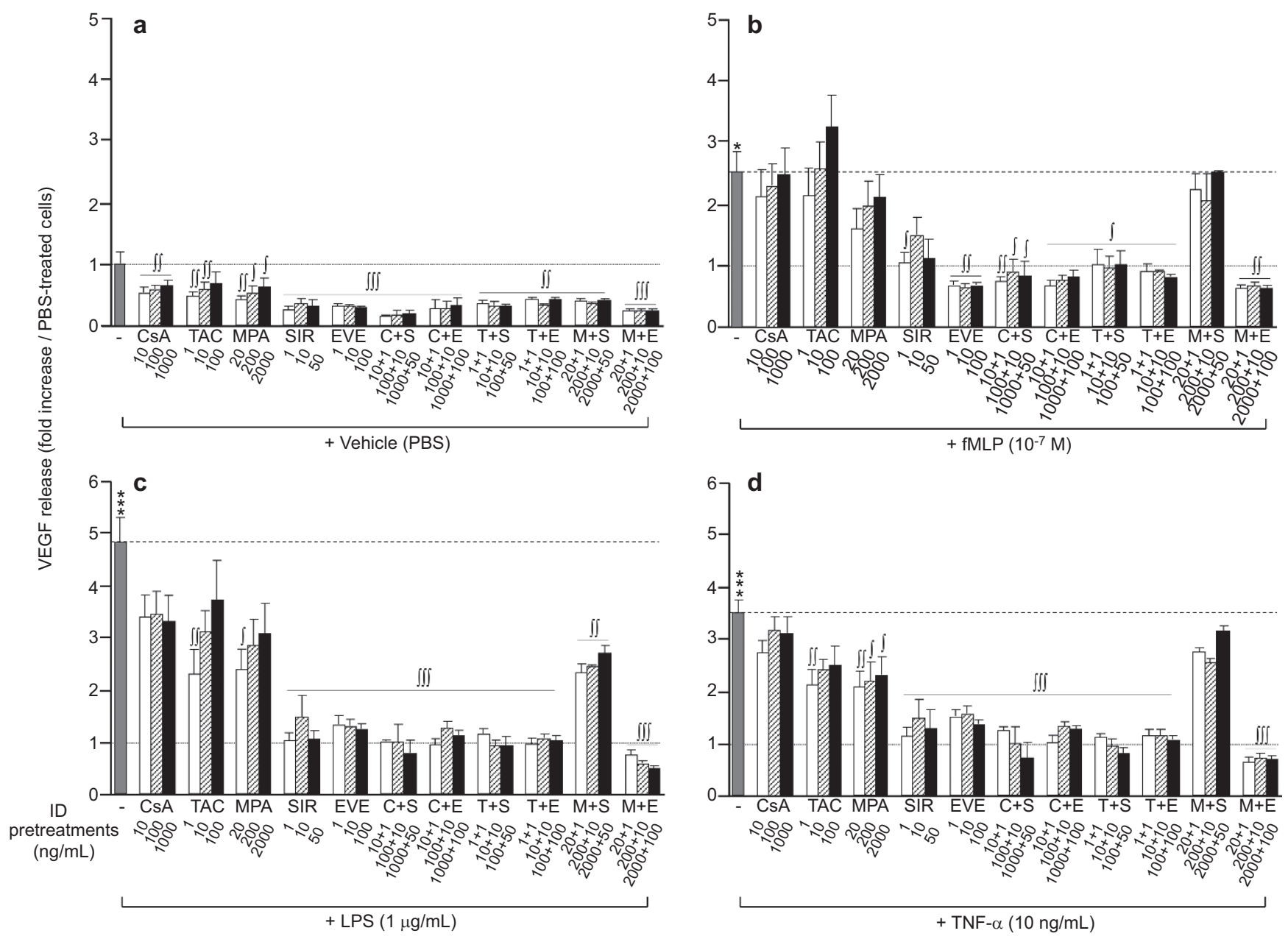

Figure 2 Effect of immunosuppressive drugs on VEGF release by neutrophils. Neutrophils were pre-treated with CsA (10, 100, 1000 ng/ml), TAC (1, 10, $100 \mathrm{ng} / \mathrm{ml}), \operatorname{MPA}(20,200,2000 \mathrm{ng} / \mathrm{ml})$, SIR $(1,10,50 \mathrm{ng} / \mathrm{ml})$ or EVE $(1,10,100 \mathrm{ng} / \mathrm{ml})$ for $30 \mathrm{~min}$ prior to agonist (fMLP, LPS or TNF- $\alpha)$ stimulation for $2 \mathrm{~h}$. VEGF was quantified by ELISA. Data are presented as the mean \pm s.e.m. of at least three independent experiments. $* P<0.05$ and $* * * P<0.001$ compared with PBS-treated neutrophils. ${ }^{\prime} P<0.05$, $\int P<0.01$; $\iint P<0.001$ compared with the respective neutrophils without ID pre-treatment. CsA, cyclosporine A; EVE, everolimus; fMLP, Formyl-Met-Leu-Phe; ID, immunosuppressive drug; LPS, lipopolysaccharide; MPA, mycophenolate acid; PBS, phosphate-buffered saline; SIR, sirolimus; TAC, tacrolimus; TNF- $\alpha$, tumor-necrosis factor- $\alpha$; VEGF, vascular endothelial growth factor.

Effect of IDs on IL-1RA release by neutrophils under proinflammatory conditions

Pre-treatment with CsA or TAC had no effect or a minor inhibitory effect on IL-1RA release by neutrophils following proinflammatory stimulation (Figure 3b-d). Pre-treatment with MPA or SIR at all concentrations had a tendency to reduce the IL-1RA release induced by fMLP, LPS and TNF- $\alpha$ (Figure $3 b-d$ ). Pre-treatment with EVE did not alter the IL-1RA release induced by fMLP (Figure 3b). However, LPS- or TNF-induced IL-1RA release was increased twofold by EVE compared with the effect of either LPS or TNF- $\alpha$ alone. Furthermore, the capacity of EVE to increase IL-1RA release was maintained even when combined with CSA, TAC or MPA during pre-incubation (Figure $3 \mathrm{c}$ and d).

Effect of IDs on IL-8 release by neutrophils under proinflammatory conditions

Pre-treatment with CsA or TAC alone had no significant effect on IL-8 release by neutrophils following pro-inflammatory stimulation (fMLP, LPS or TNF- $\alpha$ ) (Figure $4 \mathrm{~b}-\mathrm{d}$ ). Pre-treatment with MPA alone reduced the release of IL-8 upon fMLP stimulation by approximately $47 \%$ (Figure $4 \mathrm{~b}$ ). Using mTOR inhibitors, we observed an intermediate inhibitory effect of SIR on the IL-8 release evoked by fMLP $(-55 \%)$, whereas EVE completely abrogated the fMLP-mediated release of IL- 8 (Figure 4B). When SIR or EVE were combined with other IDs (CsA, TAC or MPA), SIR was no longer capable of reducing fMLP-induced IL-8 release, whereas such combinations with EVE failed to alter its capacity to reduce IL-8 release (Figure 4b). In LPS-treated neutrophils, SIR alone did not reduce IL-8 release, whereas EVE partially inhibited IL-8 release from $65-$ fold to approximately 40 -fold (Figure $4 \mathrm{c}$ ). In addition, the inhibitory capacity of EVE was retained even when combined with CsA, TAC or MPA. In contrast, SIR produced a significant inhibition of IL-8 release when combined with CsA or TAC, but not in combination with MPA (Figure 4c). Pre-treatment with EVE alone at the highest concentration reduced IL-8 

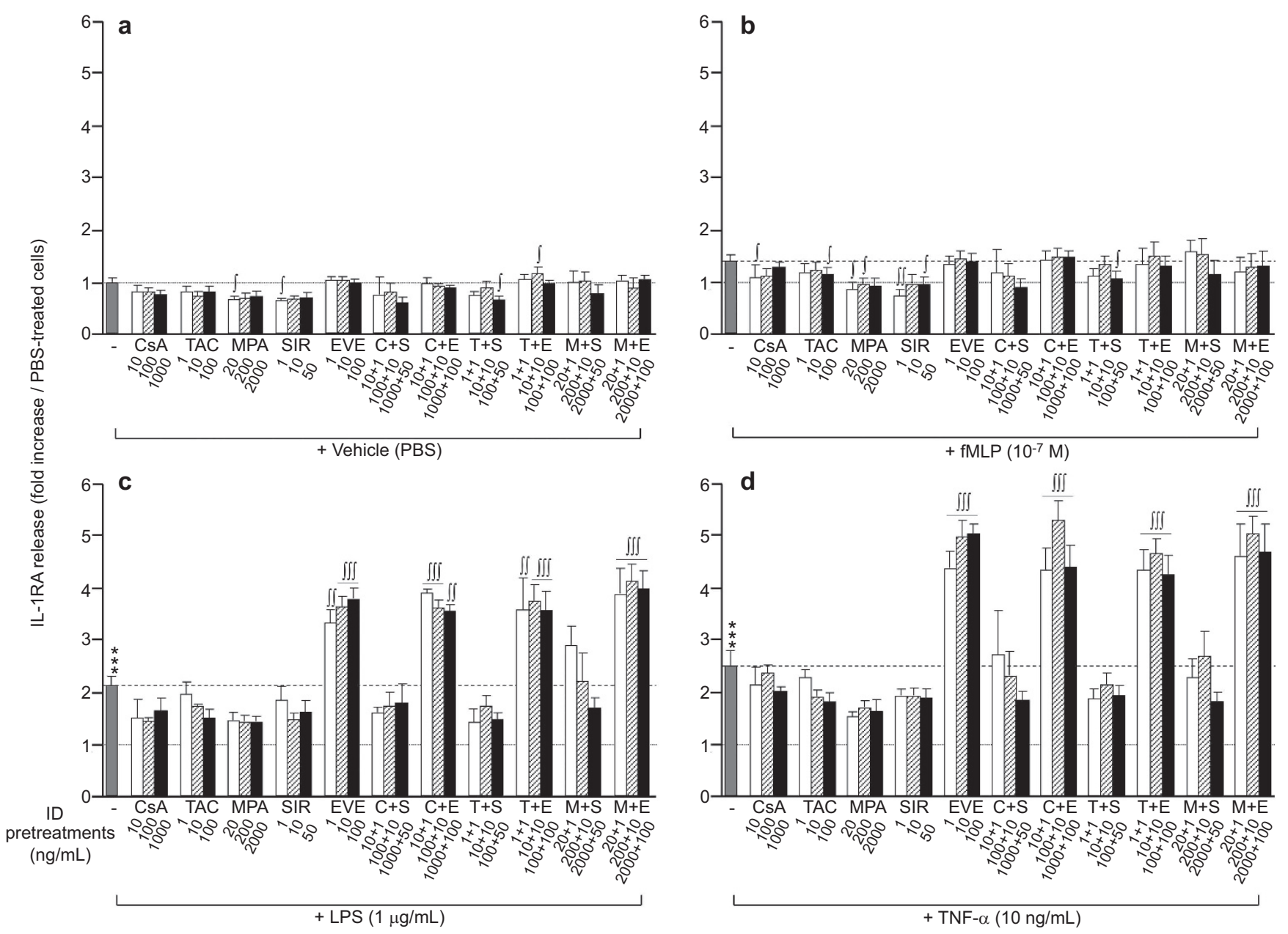

Figure 3 Effect of immunosuppressive drugs on IL-1RA release by neutrophils. Neutrophils were pre-treated with CsA (10, 100, 1000 ng/ml), TAC (1, 10, $100 \mathrm{ng} / \mathrm{ml})$, MPA (20, 200, $2000 \mathrm{ng} / \mathrm{ml})$, SIR (1, 10, $50 \mathrm{ng} / \mathrm{ml})$ or EVE (1, 10, $100 \mathrm{ng} / \mathrm{ml})$ for 30 min prior to agonist (fMLP, LPS or TNF- $\alpha)$ stimulation for $2 \mathrm{~h}$. IL-1RA release was quantified by ELISA. Data are presented as the mean \pm s.e.m. of at least three independent experiments. *** $P<0.001$ compared with PBS-treated neutrophils. ${ }^{\int} P<0.05,{ }^{\int} P<0.01 ; \iint P<0.001$ compared with the respective neutrophils without ID pretreatment. CSA, cyclosporine A; EVE, everolimus; fMLP, Formyl-Met-Leu-Phe; ID, immunosuppressive drug; IL-1RA, interleukin-1 receptor antagonist; LPS, lipopolysaccharide; MPA, mycophenolate acid; PBS, phosphate-buffered saline; SIR, sirolimus; TAC, tacrolimus; TNF- $\alpha$, tumor-necrosis factor- $\alpha$.

release following TNF- $\alpha$ stimulation (-66\%) (Figure $4 \mathrm{~d})$. Pretreatments with mTOR inhibitors (SIR or EVE) combined with CsA or TAC had the same inhibitory effect (Figure 4d). The combination of EVE with MPA induced a more potent inhibitory effect than did the combination of SIR with MPA, decreasing the release of IL- 8 following TNF- $\alpha$ stimulation by approximately $48 \%$, compared with a $16 \%$ decrease induced by SIR plus MPA (Figure 4d).

Effect of IDs on neutrophil adhesion to HUVECs and hECM We next examined the effect of IDs on neutrophil adhesion to HUVEC and hECM. For this study, we used the intermediate concentration of the different IDs used in the experiments described in Figure 4, which had a maximal or near-maximal effect on VEGF, IL-1RA or IL-8 release. Moreover, these concentrations are representative of those detected in the sera of patients having undergone an organ transplant. ${ }^{37-39}$ In basal
HBSS $^{+}$alone, neutrophil adhesion onto HUVECs was 38.3 cells $/ \mathrm{mm}^{2}$ and rose by $114 \%$ upon stimulation with TNF- $\alpha$ $(10 \mathrm{ng} / \mathrm{ml})$ (Figure 5). Pre-treatment of neutrophils with CsA, TAC, SIR or EVE had no effect (CsA, TAC, EVE) or minor inhibitory effects (SIR, $-21 \%$ ) on the basal adhesion of neutrophils to HUVECs. However, an equivalent pre-treatment with MPA did increase the basal adhesion of neutrophils onto HUVECs by $67 \%$ (Figure $5 \mathrm{a}$; left panel). In cells stimulated with TNF- $\alpha$, pre-treatment with CsA or MPA provided a slight (non-significant) increase of neutrophil adhesion to HUVECs (Figure 5a; right panel), whereas pre-treatment with TAC, SIR or EVE decreased neutrophil adhesion to HUVECs by approximately $74 \%$ (Figure $5 \mathrm{a}$; right panel).

TNF- $\alpha$ is known to promote the activation of the $\beta_{2}$-integrin (CD11b/CD18) complex on neutrophils, contributing to their adhesiveness to ECs via $\beta_{2}$-integrin binding to ICAM- 1 . We assessed whether these IDs affected neutrophil adhesion via the 

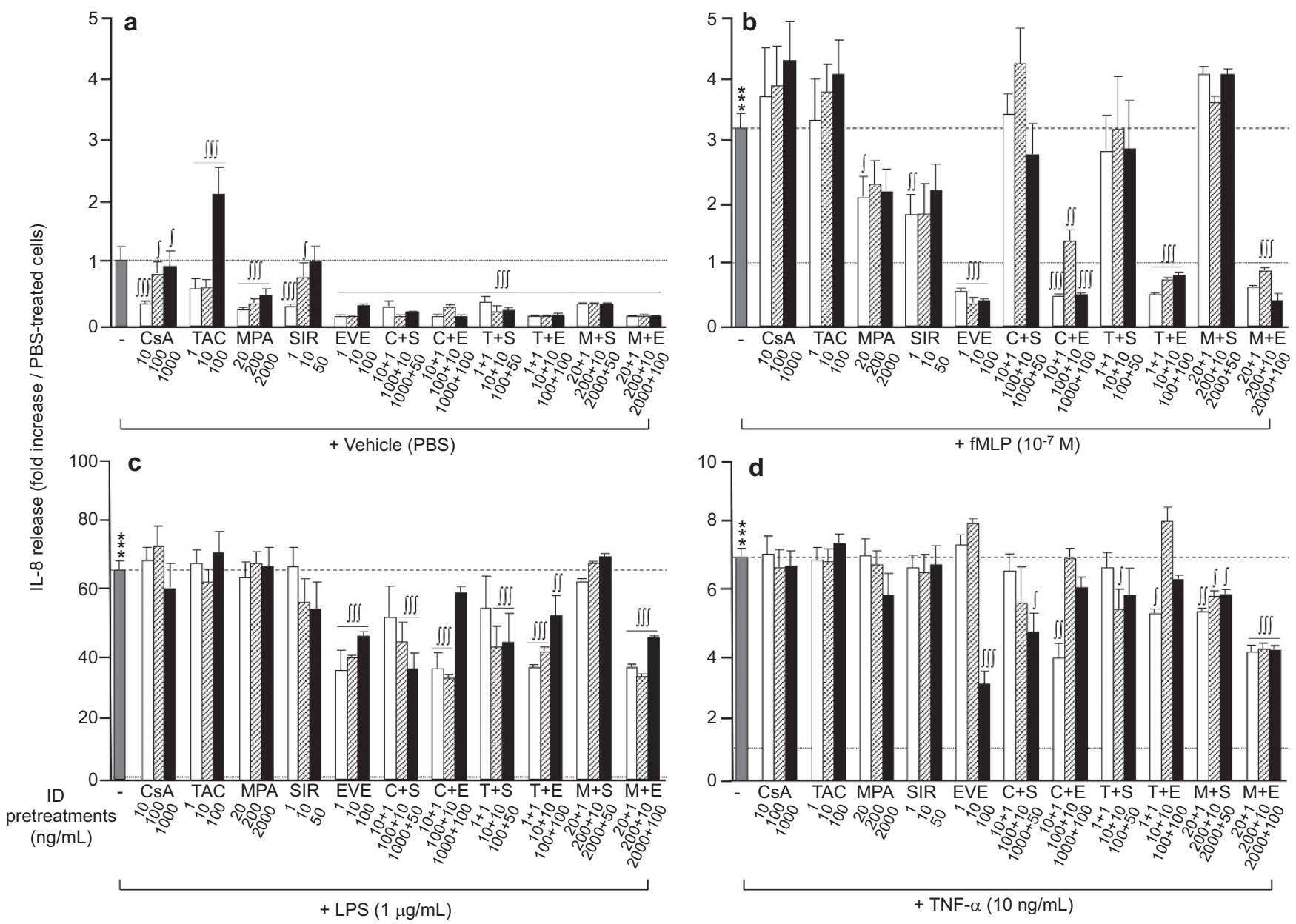

Figure 4 Effect of immunosuppressive drugs on IL-8 release by neutrophils. Neutrophils were pre-treated with CsA (10, 100, 1000 ng/ml), TAC (1, 10, $100 \mathrm{ng} / \mathrm{ml})$, MPA $(20,200,2000 \mathrm{ng} / \mathrm{ml})$, SIR $(1,10,50 \mathrm{ng} / \mathrm{ml})$ or EVE (1, 10, $100 \mathrm{ng} / \mathrm{ml})$ for 30 min prior to agonist (fMLP, LPS or TNF- $\alpha$ ) stimulation for $24 \mathrm{~h}$. IL-8 release was quantified by ELISA. Data are presented as the mean \pm s.e.m. of at least three independent experiments. $* P<0.05$ and $* * * P<0.001$ compared with PBS-treated neutrophils. $P<0.05$, $\int P<0.01$; ${ }^{\int} / \int<0.001$ compared with the respective neutrophils without ID pre-treatment. CsA, cyclosporine A; EVE, everolimus; fMLP, Formyl-Met-Leu-Phe; ID, immunosuppressive drug; IL-1RA, interleukin-1 receptor antagonist; LPS, lipopolysaccharide; MPA, mycophenolate acid; PBS, phosphate-buffered saline; SIR, sirolimus; TAC, tacrolimus; TNF- $\alpha$, tumor-necrosis factor- $\alpha$.

modulation of CD11b/CD18 activation. Pre-treatment of neutrophils with an antibody against human $\beta_{2}$-integrin/CD18 did not affect their adhesion to HUVECs either under basal$\mathrm{HBSS}^{+}$conditions or when pre-treated with CsA, TAC, SIR or EVE. However, blocking human $\beta_{2}$-integrin/CD18 did prevent an increase in neutrophil adhesion induced by MPA (Figure 5a; left panel). Furthermore, blocking human $\beta_{2}$-integrin/CD18 prevented the increase of neutrophil adhesion induced by TNF- $\alpha$ (Figure 5a; right panel) in the presence or absence of immunosuppressive drugs.

The combination of IDs had no effect or minor effects (slight reduction) on the basal adhesion of neutrophils to HUVECs. In addition, the effect of MPA alone was prevented by its combination with TAC, SIR, EVE and only in part by CsA (Figure $5 \mathrm{~b}$; left panel). Blocking $\beta_{2}$-integrin/CD18 in conjunction with the addition of combinations of IDs tended to reduce neutrophil adhesiveness to levels slightly below those observed under basal $\mathrm{HBSS}^{+}$conditions (Figure 5b, left panel). Pretreatment of neutrophils with a combination of IDs prior to TNF- $\alpha$ stimulation failed to augment the effects of TNF- $\alpha$ on neutrophil adhesion to HUVECs (Figure $5 \mathrm{~b}$; right panel). The combination of TAC with mTOR inhibitors (EVE or SIR) prevented the increase of neutrophil adhesion induced by TNF- $\alpha$ by $96 \%$ and $80 \%$, respectively. The results obtained with other combinations did not reach statistical significance, but the combination of CsA + SIR and MPA+EVE displayed a trend to reduce the adhesion of neutrophils by $46 \%(P=0.08)$ and $53 \%(P=0.09)$ (Figure 5b; right panel). As observed with IDs administered individually (Figure 5a; right panel), blocking $\beta_{2}$-integrin/CD18 in the presence or absence of various combinations of IDs abrogated the ability of TNF- $\alpha$ to increase neutrophil adhesion (Figure 5b; right panel).

To assess the effects of IDs on the adhesiveness of activated neutrophils independently from the activation of endothelial 

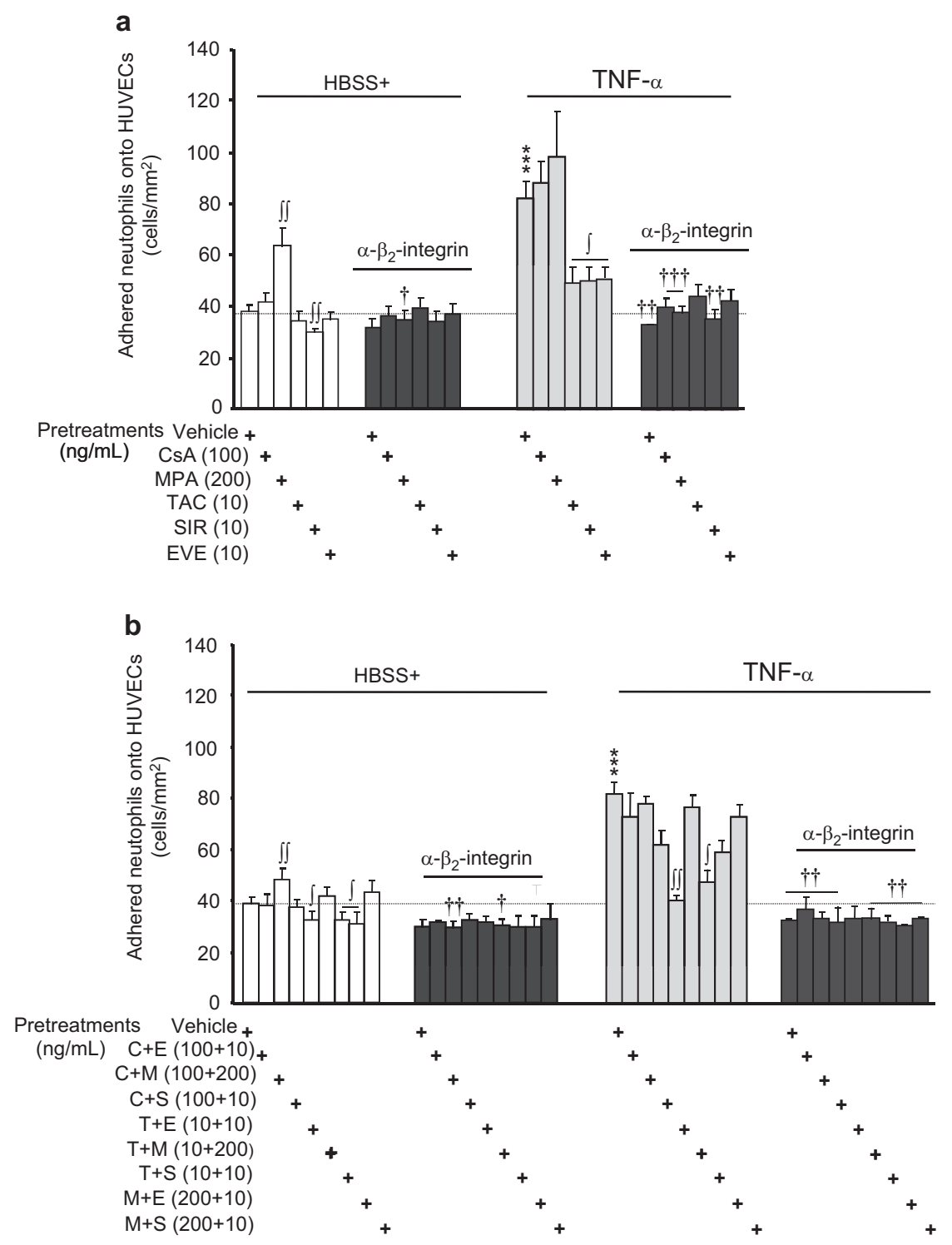

Figure 5 Effect of immunosuppressive drugs on neutrophil adhesion to HUVECs. Neutrophils were pre-treated with or without the indicated immunosuppressive drugs (30 min) alone (a) or in combination (b). A blocking human $\beta_{2}$-integrin/CD18 antibody (2 $\left.\mu \mathrm{g} / \mathrm{ml}\right) \mathrm{was}$ added to neutrophils $15 \mathrm{~min}$ prior to the addition of PBS or TNF- $\alpha(10 \mathrm{ng} / \mathrm{ml})$ for $7.5 \mathrm{~min}$. Upon the adhesion of neutrophils to HUVECs or hECM, the wells were gently rinsed with PBS to remove non-adherent neutrophils, and adhered neutrophils were fixed with a $1 \%$ paraformaldehyde/1\% glutaraldehyde-PBS solution. For each well, four fields of view by microscopy were randomly selected, and the neutrophils in each field of view were counted and recorded as the number of adhered neutrophils $/ \mathrm{mm}^{2}$. Data are presented as the mean \pm s.e. $\mathrm{m}$. of 16 independent experiments. $* * * P<0.001$ compared to PBS-treated neutrophils. ${ }^{P}<0.05$; $\int P<0.01$ compared with the respective neutrophils without ID pre-treatment. ${ }^{\dagger} P<0.05 ;{ }^{\dagger} P<0.01 ;{ }^{\dagger \dagger} P<0.001$ compared with neutrophils without the blocking human $\beta_{2}$-integrin/CD18 antibody. hECM, human extracellular matrix; ID, immunosuppressive drug; PBS, phosphate-buffered saline; TNF- $\alpha$, tumor-necrosis factor- $\alpha$.

cells, we used hECM as a binding substrate. Under basal $\left(\mathrm{HBSS}^{+}\right.$) conditions, the adhesion of neutrophils onto hECM was 27.6 cells $/ \mathrm{mm}^{2}$ and rose by $175 \%$ following stimulation with TNF- $\alpha$ (10 ng/ml; Figure 6). Pre-treatment of neutrophils with CsA, TAC, SIR or EVE had no effect on the basal adhesion of neutrophils onto hECM. However, as observed when using HUVECs (Figure 5), pre-treatment with MPA activated neutrophils and increased their adhesion onto hECM by $80 \%$ (Figure 6a; left panel). In contrast to its effects under basal conditions, pre-treatment with CsA increased neutrophil adhesion in cells treated with TNF- $\alpha$ (294\% over $\mathrm{HBSS}^{+}$and $43 \%$ over TNF- $\alpha$; Figure $6 \mathrm{a}$, right panel). However, pre-treatment with MPA had no significant effect, whereas pre-treatment with TAC, SIR or EVE reduced TNF- $\alpha$-mediated neutrophil adhesion onto hECM by $65 \%, 80 \%$ and $60 \%$, respectively (Figure $6 a$; right panel).

Blocking $\beta_{2}$-integrin/CD18 in neutrophil preparations under basal-HBSS $^{+}$conditions and in the presence or absence of IDs reduced the adhesion of neutrophils to hECM by $\sim 48 \%$ (Figure 6a; left panel). In addition, pre-treatment with a 

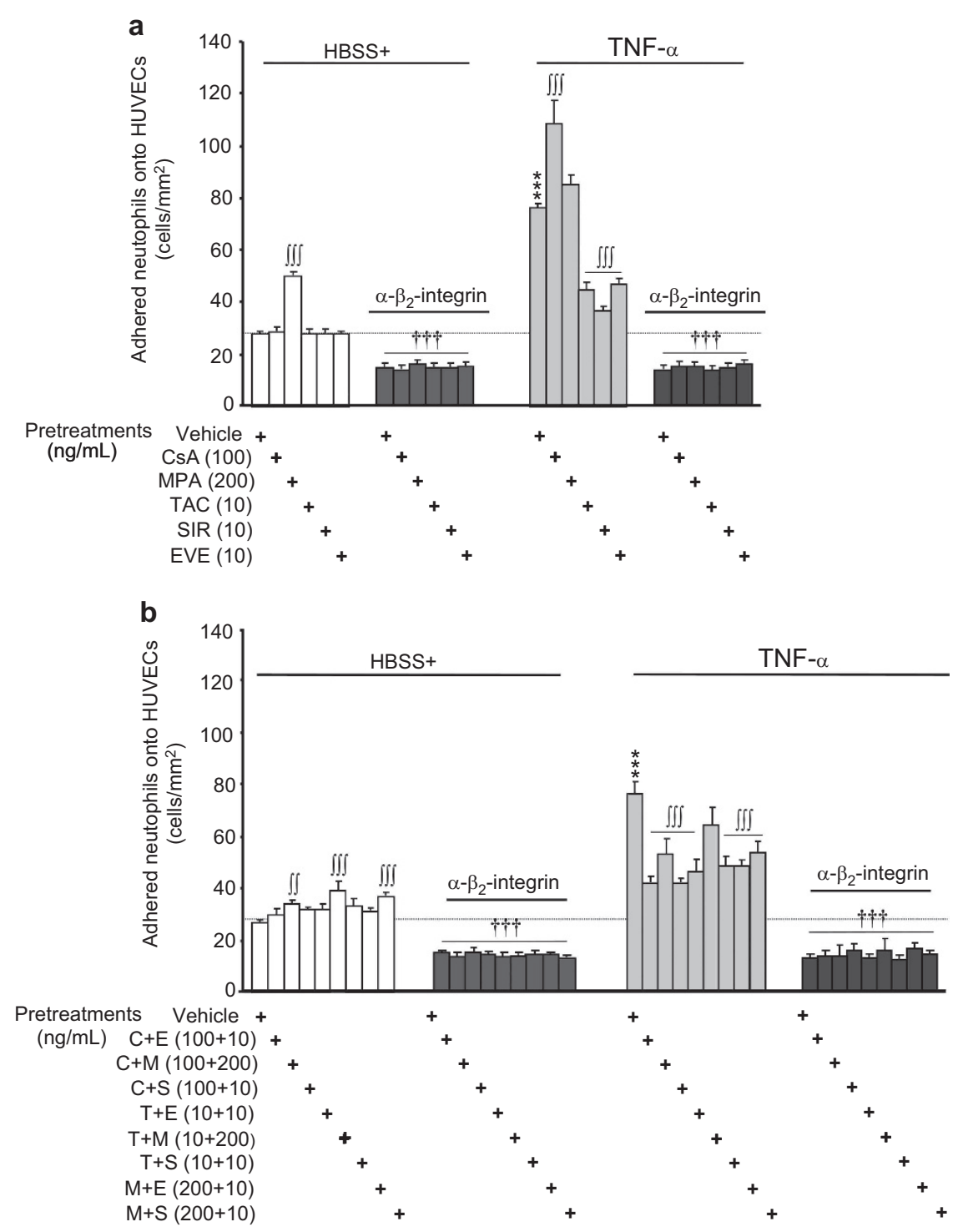

Figure 6 Effect of immunosuppressive drugs on neutrophil adhesion to hECM. Neutrophils were pre-treated with or without the indicated immunosuppressive drugs (30 min) alone (a) or in combination (b), and blocking human $\beta_{2}$-integrin/CD18 antibody ( $\left.2 \mu \mathrm{g} / \mathrm{ml}\right)$ was added to hECM 15 min prior to the addition of PBS or TNF- $\alpha(10 \mathrm{ng} / \mathrm{ml})$ for $7.5 \mathrm{~min}$. Data are presented as the mean \pm s.e.m. of eight independent experiments. ${ }^{* * *} P<0.001$ compared with PBS-treated neutrophils. "I $P<0.01$; $\iint \mid P<0.001$ compared with the respective neutrophils without ID pre-treatment. ${ }^{\dagger \dagger} P<0.001$ compared with neutrophils without the blocking human $\beta_{2}$-integrin/CD18 antibody. hECM, human extracellular matrix; ID, immunosuppressive drug; PBS, phosphate-buffered saline; TNF- $\alpha$, tumor-necrosis factor- $\alpha$.

blocking human $\beta_{2}$-integrin/CD18 antibody completely prevented the ability of TNF- $\alpha$ to increase neutrophil adhesion onto hECM. Furthermore, we observed that certain IDs were able to reduce the ability of TNF- $\alpha$ to increase neutrophil adhesion and that the residual binding of neutrophils to hECM was eliminated upon blocking $\beta_{2}$-integrin/CD18 (Figure 6a; right panel).

In general, we observed that the combination of IDs either lacked any effect or enhanced (up to $+47 \%$; TAC + MPA) the basal adhesion of neutrophils onto hECM (Figure 6b; left panel). In each case, the addition of a blocking human $\beta_{2}$-integrin/ CD18 antibody reduced the basal adhesion of neutrophils to $\sim 50 \%$ of that observed under the basal $\mathrm{HBSS}^{+}$condition
(Figure 6b; left panel). Pre-treatment with all of the combinations of IDs prior to TNF- $\alpha$ stimulation (except for TAC+MPA; $-25 \%)$ significantly reduced the adhesion of neutrophils onto hECM (by 47\%-71\%), (Figure 6b; right panel). Finally, blocking $\beta_{2}$-integrin/CD18 maintained neutrophil adhesion at levels comparable to those of control $\mathrm{HBSS}^{+}$-treated neutrophils, even when the cells were pre-treated with various combinations of IDs followed by TNF- $\alpha$ (Figure 6b; right panel).

\section{DISCUSSION}

In the present study, we report that neutrophils isolated from the venous blood of healthy volunteers are able to release IL-8, VEGF and IL-1RA in response to a pro-inflammatory stimulus. 
Using different IDs alone or combined, as routinely given to heart transplant recipients, we observed that mTOR inhibitors, namely, EVE and SIR, were the most efficient IDs and had maximal or near maximal inhibitory effects on the neutrophils' pro-inflammatory response. Globally, EVE was more efficient than SIR alone to decrease IL-8 and VEGF release from neutrophils. Notably, EVE was the only ID capable of promoting the release of the anti-inflammatory cytokine IL-1RA. Additionally, this latter effect of EVE was maintained when combined to other IDs such as CsA, TAC or MPA. Finally, we report that in response to a pro-inflammatory stimulation $(\mathrm{TNF}-\alpha)$, pre-treatment with TAC, EVE or SIR, alone or in combination, decreased the activation of the $\beta_{2}$-integrin complex (CD11b/CD18), resulting in reduced neutrophil adhesiveness to HUVECs and hECM.

CTX remains the predominant treatment for patients suffering from end stage heart failure. ${ }^{42}$ Despite the improvement of immunosuppressive therapies, the first cause of mortality at 5 years following CTX remains the development of CAV, ${ }^{1}$ and the rate of late rejection remains unchanged, achieving an approximately $50 \%$ survival rate at 10 years post-transplant. ${ }^{43}$ Although the pathological changes of CAV are well documented, ${ }^{1,2}$ its etiology remains unclear, and the intimate mechanisms underlying the development of this condition are not fully understood. Investigative efforts have focused primarily on the acquired immune system, particularly on the involvement of lymphocytes, ${ }^{44,45}$ with less attention given to the impact of innate immune cells. Previous animal studies demonstrated the potential deleterious effect of neutrophil graft infiltration ${ }^{46}$ and neutrophil myocardial accumulation via the participation of activated integrins upon heart transplantation. ${ }^{13,14}$ However, though neutrophils are able to release pro-inflammatory mediators, no studies have determined the potential role of neutrophil-mediated inflammation in the development of CAV in humans. Thus, we aimed to assess the impact of different ID regimens on the inflammatory response of neutrophils isolated from venous blood of healthy volunteers. First, we report that neutrophils are able to release different mediators such as VEGF, IL-1RA and IL-8 in response to pro-inflammatory stimulation, which is consistent with previous reports. ${ }^{10,15}$ In our study, LPS and TNF- $\alpha$ were the two most potent agonists in triggering the release of the three cytokines.

\section{Effect of immunosuppressive drugs on the release of cytokines by human neutrophils}

Over the last 30 years, immunosuppressive therapies have been the gold standard approach to prevent organ rejection in heart transplant recipients. ${ }^{47}$ Although the use of IDs has led to an improvement in the survival rates after $\mathrm{CTX},{ }^{48}$ their beneficial outcome is plagued by adverse side effects. For instance, some studies have reported inefficient or worsening renal function induced by CsA treatment, ${ }^{49,50}$ while other groups have observed negative effects of MPA and TAC by inducing endothelial cell dysfunction and altering vasorelaxation properties. ${ }^{51-53}$ However, still other studies have reported beneficial effects of mTOR inhibitors, including the capacity of SIR to decrease long-term CAV progression ${ }^{26}$ and EVE to delay or prevent CAV progression. ${ }^{27,28}$ In the present study, we demonstrate that under basal conditions, all immunosuppressive drugs (CsA, TAC, MPA, SIR and EVE) reduced the release of pro-inflammatory mediators (VEGF and IL-8) without affecting the basal release of the anti-inflammatory cytokine IL-1RA. Under basal (PBS) and pro-inflammatory (fMLP, LPS or TNFa) conditions, mTOR inhibitors, alone or in combination, were the most efficient at decreasing VEGF and IL-8 release.

Although SIR and EVE appear to be the most efficient IDs at preventing the release of pro-inflammatory cytokines in response to TNF- $\alpha$, EVE showed a more prominent protective effect than did SIR. For instance, EVE was able to completely abrogate the release of VEGF and IL- 8 by the neutrophils under fMLP stimulation while also being the most efficient at preventing an increase in IL-8 release in response to LPS stimulation. In addition, while EVE (alone) provided an anti-inflammatory activity via the inhibition of VEGF and IL-8 release, it also promoted the induction of IL-1RA release, and this effect was maintained when combined with any of the other IDs examined in the present study. Under the same conditions, SIR was not as efficient as EVE when combined with other IDs. For instance, when combined with MPA, SIR was either equally or less efficient in preventing VEGF release in response to pro-inflammatory stimulation. Notably, EVE was able to further increase the release of IL-1RA from neutrophils during stimulation with LPS or TNF- $\alpha$. Our data are consistent with recent studies reporting that SIR is more efficient than calcineurin inhibitors (CsA and TAC) at decreasing the release of pro-inflammatory mediators by neutrophils from healthy volunteers under pro-inflammatory conditions. ${ }^{54}$

The mTOR pathway (phosphatidylinositol 3-kinase-AktmTOR) is a critical signaling pathway that is sensitive to input from multiple signals (e.g., growth factors, hormones, nutrients, cytokines and stress) and responds by specifically enhancing the translation of messenger RNAs encoding key proteins. ${ }^{55,56}$ Previous studies have reported that the two best characterized pathways downstream of mTOR activation are mediated by ribosomal protein $\mathrm{S} 6$ kinase and by eukaryotic initiation factor binding protein, which participate in mRNA translation into corresponding proteins. ${ }^{57}$ The inhibitors of mTOR, including EVE (or analogs), may inhibit the translation machinery directly or through unknown mechanisms. ${ }^{58}$ In the latter study, the authors reported that EVE increases the basal release of multiple cytokines in mouse macrophages (e.g., IL-6, MCP-1, Rantes and TNF- $\alpha$ ). The authors also reported that the inhibition of protein translation induces p38 MAPK activation, thus leading to cytokine synthesis and release. In a recent study, we observed that LPS and angiopoietin-1 are both capable of increasing IL-1RA mRNA transcription in human neutrophils without promoting its de novo protein synthesis. In addition, we observed that human neutrophils have a high level of constitutive IL-1RA protein content and that stimulation with either LPS or angiopoietin-1 induces approximately $10 \%$ of the total endogenous content of the IL-1RA protein. ${ }^{59}$ Our data suggest 
that under basal condition, EVE is not sufficient to trigger the basal release of IL-1RA from human neutrophils; however, under pro-inflammatory stimuli (e.g., LPS or TNF- $\alpha$ ), it appears sufficient to potentiate IL-1RA release, and this latter effect seems to be specific to EVE compared with SIR. The mechanism underlying the discrepancy between these mTOR inhibitors remains to be elucidated.

\section{Effect of immunosuppressive drugs on human neutrophil activation and adhesion}

Other studies have explored the effects of IDs on neutrophil activation, adhesion and/or migration. Treatment of human neutrophils with SIR and TAC inhibits granulocyte-macrophage colony-stimulating factor and IL-8-mediated chemotactic activity ${ }^{60,61}$ In mice, SIR decreased Toll-like receptor-2- and -4 -induced neutrophil activation. ${ }^{62}$

In the present study, we examined the acute/immediate effect of IDs on neutrophil activation. Specifically, we assessed the formation of an active CD11b/CD18 complex that binds to its counter-receptor ligand, ICAM-1, which is constitutively expressed on endothelial cells. ${ }^{63}$ Following TNF- $\alpha$ stimulation, we observed a marked increase in neutrophil adhesion to HUVECs that was completely abrogated (back to basal levels) when neutrophils were pre-treated with an antibody that blocks $\beta_{2}$-integrin/CD18. These findings demonstrate that the active $\mathrm{CD} 11 \mathrm{~b} / \mathrm{CD} 18$ complex is essential for the acute/ immediate adhesion of neutrophils to HUVECs under proinflammatory conditions, which is in agreement with previous reports. ${ }^{64,65}$ By pre-treating neutrophils with IDs, we were able to assess their relative effects on CD11b/CD18 complex activation and neutrophil adhesion. Our data demonstrated that under basal conditions, all of the IDs tested (with the exception of MPA) failed to induce either the adhesion of neutrophils onto HUVECs or the activation of the CD11b/CD18 complex. The ability of MPA to promote neutrophil adhesion to HUVECs was abrogated by blocking $\beta_{2}$-integrin/CD18, demonstrating that MPA alone can induce acute/immediate neutrophil activation. Pre-treatment with TAC, SIR or EVE reduced (by approximately 70\%) the TNF- $\alpha$ induced adhesion of neutrophils to HUVECs, whereas CsA or MPA had no effect. Blocking $\beta_{2}$-integrin/CD18 fully abrogated TNF- $\alpha$-mediated neutrophil activation in the presence of CsA or MPA and completed the inhibitory effect of TAC, SIR and EVE. Taken together, these data demonstrate the capacity of TAC, SIR and EVE to reduce TNF- $\alpha$-mediated neutrophil activation. Even when administered in various combinations, these three IDs maintained their capacity to prevent neutrophil adhesion onto HUVECs and activation of the CD11b/CD18 complex under basal and TNF- $\alpha$-stimulated conditions.

Upon activation, P-selectin translocates to the surface of endothelial cells. P-selectin is the ligand for P-selectin glycoprotein, which is constitutively expressed on neutrophils. ${ }^{66}$ To confirm the direct effect of IDs on neutrophils and exclude the potential contribution of endothelial cell activation, we assessed the binding of neutrophils onto hECM. Under nonstimulated conditions, we observed a basal level of neutrophil adhesion onto hECM; this level was partly reduced upon blocking $\beta_{2}$-integrin/CD18, suggesting a basal level of CD11b/CD18 activation. Upon the addition of IDs, we essentially observed the same pattern of effects upon adhesion as observed in the presence of HUVECs. Only MPA promoted the adhesion of neutrophils onto hECM under basal conditions, and this effect was abrogated in upon blocking $\beta_{2}$-integrin/CD18. These results confirm that MPA is able to activate neutrophils. These data are consistent with a previous study demonstrating that MPA induces a chronic increase in the expression of vascular cell adhesion molecule-1 and E-selectin on HUVECs. ${ }^{67}$ Furthermore, we observed that TAC, SIR and EVE were all capable of reducing TNF- $\alpha$-induced neutrophil adhesion to hECM. In contrast, CsA or MPA were unable to decrease neutrophil adhesion. In each case, blocking $\beta_{2}$-integrin/CD18 suppressed the adhesion of neutrophils onto hECM to the levels detected under basal conditions. Finally, as observed in our studies of neutrophil adhesion to HUVECs, binary combinations of TAC, SIR and EVE proved to be the most effective at reducing $\mathrm{CD} 11 \mathrm{~b} / \mathrm{CD} 18$ complex activation and the resulting adhesion of neutrophils to hECM.

In summary, our study demonstrates that TAC, SIR and EVE are the most effective immunosuppressive drugs at reducing $\mathrm{CD} 11 \mathrm{~b} / \mathrm{CD} 18$ complex activation and the corresponding neutrophil adhesion to hECM or endothelial cells. In addition, we observed that mTOR inhibitors (SIR and EVE) were the most effective at preventing the release of pro-inflammatory mediators (VEGF and IL-8) by neutrophils. Notably, only the new generation mTOR inhibitor, EVE, promoted the release of the anti-inflammatory cytokine IL-1RA. It is also important to note that in our studies, when EVE was combined with different IDs, it retained its anti-inflammatory activities. This was not always the case for SIR. These studies investigated neutrophils isolated from healthy volunteers, and the clinical impact of our observations thus needs to be validated in patients following organ transplantation.

\section{ACKNOWLEDGEMENTS}

This work was supported by the Carolyn and Richard Renaud Research Chair in Heart Failure of the Montreal Heart Institute. The authors are thankful to all of their volunteers for kindly providing us with blood samples. The authors are grateful to the superb work provided by the secretarial team and the nurses at the Montreal Heart Institute.

1 Schmauss D, Weis M. Cardiac allograft vasculopathy: recent developments. Circulation 2008; 117: 2131-2141.

2 Colvin-Adams M, Agnihotri A. Cardiac allograft vasculopathy: current knowledge and future direction. Clin Transplant2011; 25: 175-184.

3 Rahmani M, Cruz RP, Granville DJ, McManus BM. Allograft vasculopathy versus atherosclerosis. Circ Res 2006; 99: 801-815.

4 Valantine HA. Cardiac allograft vasculopathy: central role of endothelial injury leading to transplant "atheroma". Transplantation 2003; 76: 891-899.

5 Behrendt D, Ganz P, Fang JC. Cardiac allograft vasculopathy. Curr Opin Cardiol 2000; 15: 422-429. 
6 Blakely ML, Van der Werf WJ, Berndt MC, Dalmasso AP, Bach FH \& Hancock WW. Activation of intragraft endothelial and mononuclear cells during discordant xenograft rejection. Transplantation 1994; 58: 1059-1066.

7 Zhang XP, Kelemen SE, Eisen HJ. Quantitative assessment of cell adhesion molecule gene expression in endomyocardial biopsy specimens from cardiac transplant recipients using competitive polymerase chain reaction. Transplantation 2000; 70: 505-513.

8 Mancini MC, Evans JT. Role of platelet-derived growth factor in allograft vasculopathy. Ann Surg 2000; 231: 682-688.

9 van Loosdregt J, van Oosterhout MF, Bruggink AH, van Wichen DF, van Kuik J, de Koning $\mathrm{E}$ et al. The chemokine and chemokine receptor profile of infiltrating cells in the wall of arteries with cardiac allograft vasculopathy is indicative of a memory T-helper 1 response. Circulation 2006; 114: 1599-1607.

10 Mantovani A, Cassatella MA, Costantini C, Jaillon S. Neutrophils in the activation and regulation of innate and adaptive immunity. Nat Rev Immunol 2011; 11: 519-531.

11 Nathan C. Neutrophils and immunity: challenges and opportunities. Nat Rev Immunol 2006; 6: 173-182.

12 Phillipson $M$, Kubes $P$. The neutrophil in vascular inflammation. Nat Med 2011; 17: 1381-1390.

13 Oubenaissa $A$ et al. Evidence for an involvement of the neutrophil integrin lymphocyte function-associated antigen-1 in early failure of heart transplants. Circulation 1996; 94: II254-II259.

14 Shimizu K, Libby P, Shubiki R, Sakuma M, Wang Y, Asano K et al. Leukocyte integrin Mac-1 promotes acute cardiac allograft rejection. Circulation 2008; 117, 1997-2008.

15 Neagoe PE, Dumas E, Hajjar F, Sirois MG. Angiopoietin-1 but not angiopoietin-2 induces IL-8 synthesis and release by human neutrophils. J Cell Physiol 2012; 227: 3099-3110.

16 White M, Ross H, Haddad H, LeBlanc MH, Racine N, Pflugfelder P et al. Subclinical inflammation and prothrombotic state in heart transplant recipients: impact of cyclosporin microemulsion vs. tacrolimus. Transplantation 2006; 82: 763-770.

17 el-Sawy T, Fahmy NM, Fairchild RL. Chemokines: directing leukocyte infiltration into allografts. Curr Opin Immunol 2002; 14: 562 568.

18 Morita K, Miura M, Paolone DR, Engeman TM, Kapoor A, Remick DG et al. Early chemokine cascades in murine cardiac grafts regulate $T$ cell recruitment and progression of acute allograft rejection. J Immunol 2001; 167: 2979-2984.

19 Kao J, Kobashigawa J, Fishbein MC, MacLellan WR, Burdick MD, Belperio JA et al. Elevated serum levels of the CXCR3 chemokine ITAC are associated with the development of transplant coronary artery disease. Circulation 2003; 107: 1958-1961.

20 Raichlin ER, McConnell JP, Lerman A, Kremers WK, Edwards BS, Kushwaha SS et al. Systemic inflammation and metabolic syndrome in cardiac allograft vasculopathy. J Heart Lung Transplant 2007; 26: 826-833.

21 Badiwala MV, Guha D, Tumiati L, Joseph J, Ghashghai A, Ross HJ et al. Epidermal growth factor-like domain 7 is a novel inhibitor of neutrophil adhesion to coronary artery endothelial cells injured by calcineurin inhibition. Circulation 2011; 124: S197-S203.

22 Li H, Tanaka K, Chhabra A, Oeser B, Kobashigawa JA \& Tobis JM et al. Vascular remodeling 1 year after cardiac transplantation. J Heart Lung Transplant 2007; 26: 56-62.

23 Dandel M, Jasaityte R, Lehmkuhl H, Knosalla C, Hetzer R. Maintenance immunosuppression with mycophenolate mofetil: long-term efficacy and safety after heart transplantation. Transplant Proc 2009; 41: 2585-2588.

24 von Vietinghoff S, Ouyang H, Ley K. Mycophenolic acid suppresses granulopoiesis by inhibition of interleukin-17 production. Kidney Int 2010; 78: 79-88.

25 Feterowski C, Weighardt H, Emmanuilidis K, Hartung T, Holzmann B. Immune protection against septic peritonitis in endotoxin-primed mice is related to reduced neutrophil apoptosis. Eur J Immunol 2001; 31: 1268-1277.

26 Topilsky Y, Hasin T, Raichlin E, Boilson BA, Schirger JA, Pereira NL et al. Sirolimus as primary immunosuppression attenuates allograft vasculopathy with improved late survival and decreased cardiac events after cardiac transplantation. Circulation 2012; 125: 708720.

27 Eisen HJ, Tuzcu EM, Dorent R, Kobashigawa J, Mancini D, Valantinevon Kaeppler HA et al. Everolimus for the prevention of allograft rejection and vasculopathy in cardiac-transplant recipients. $N$ Engl J Med 2003; 349: 847-858.

28 Ishida J, Kinugawa K, Shiga T, Imamura T, Hatano M, Maki H et al. Rapidly progressive cardiac allograft vasculopathy in early onset regressed with everolimus treatment in an adult cardiac recipient. Int Heart J 2012; 53: 388-390.

29 Schweiger M, Stiegler P, Puntschart A, Sereinigg M, Prenner G, Wasler $A$ et al. Everolimus in different combinations as maintenance immunosuppressive therapy in heart transplant recipients. Exp Clin Transplant 2012; 10: 273-277.

30 Thibodeau JT, Mishkin JD, Patel PC, Kaiser PA, Ayers CR, Mammen $\mathrm{PP}$ et al. Sirolimus use and incidence of venous thromboembolism in cardiac transplant recipients. Clin Transplant 2012; 26: 953-959.

31 Arora S, Erikstad I, Ueland T, Sigurdardottir V, Ekmehag B, Jansson K et al. Virtual histology assessment of cardiac allograft vasculopathy following introduction of everolimus-results of a multicenter trial. Am J Transplant 2012; 12: 2700-2709.

32 Brkovic A, Pelletier M, Girard D, Sirois MG. Angiopoietin chemotactic activities on neutrophils are regulated by PI-3K activation. J Leukoc Biol 2007; 81: 1093-1101.

33 Neagoe PE, Brkovic A, Hajjar F, Sirois MG. Expression and release of angiopoietin-1 from human neutrophils: intracellular mechanisms. Growth Factors 2009; 27: 335-344.

34 Cassatella MA, Bazzoni F, Ceska M, Ferro I, Baggiolini M \& Berton G et al. IL-8 production by human polymorphonuclear leukocytes. The chemoattractant formyl-methionyl-leucyl-phenylalanine induces the gene expression and release of IL-8 through a pertussis toxin-sensitive pathway. J Immunol 1992; 148: 3216-3220.

35 Fujishima S, Hoffman AR, Vu T, Kim KJ, Zheng H, Daniel D et al. Regulation of neutrophil interleukin 8 gene expression and protein secretion by LPS, TNF-alpha, and IL-1 beta. J Cell Physiol 1993; 154: 478-485.

36 Malyak M, Smith MF Jr, Abel AA, Arend WP. Peripheral blood neutrophil production of interleukin-1 receptor antagonist and interleukin-1 beta. J Clinical Immunol 1994; 14: 20-30.

37 Fuchs U, Zittermann A, Hakim-Meibodi K, Borgermann J, Schulz U \& Gummert JF et al. Everolimus plus dosage reduction of cyclosporine in cardiac transplant recipients with chronic kidney disease: a twoyear follow-up study. Transplant Proc 2011; 43: 1839-1846.

38 Kahan BD, Keown P, Levy GA \& Johnston A. Therapeutic drug monitoring of immunosuppressant drugs in clinical practice. Clin Therap 2002; 24: 330-350; discussion 329.

39 Wong SH. Therapeutic drug monitoring for immunosuppressants. Clin Chim Acta 2001; 313: 241-253.

40 Lemieux C, Maliba R, Favier J, Theoret JF, Merhi Y \& Sirois MG et al. Angiopoietins can directly activate endothelial cells and neutrophils to promote proinflammatory responses. Blood 2005; 105: 15231530.

41 Baines KJ, Simpson JL, Scott RJ, Gibson PG. Immune responses of airway neutrophils are impaired in asthma. Exp Lung Res 2009; 35: 554-569.

42 Koerner MM, Durand JB, Lafuente JA, Noon GP, Torre-Amione G. Cardiac transplantation: the final therapeutic option for the treatment of heart failure. Curr Opin Cardiol 2000; 15: 178-182.

43 Hertz MI, Aurora P, Christie JD, Dobbels F, Edwards LB, Kirk R et al. Registry of the International Society for Heart and Lung Transplantation: a quarter century of thoracic transplantation. J Heart Lung Transplant 2008; 27: 937-942.

44 Methe H, Wiegand D, Welsch U, Krombach F, Meiser B, Nabauer M et al. Peripheral expansion of circulating T-helper 1 cells predicts coronary endothelial dysfunction after cardiac transplantation. J Heart Lung Transplant 2005; 24: 833-840.

45 Motallebzadeh R, Rehakova S, Conlon TM, Win TS, Callaghan CJ, Goddard $\mathrm{M}$ et al. Blocking lymphotoxin signaling abrogates the 
development of ectopic lymphoid tissue within cardiac allografts and inhibits effector antibody responses. FASEB J 2012; 26: 51-62.

46 Akimoto H, McDonald TO, Weyhrich JT, Thomas R, Rothnie CL \& Allen $\mathrm{MD}$ et al. Antibody to CD18 reduces neutrophil and T lymphocyte infiltration and vascular cell adhesion molecule- 1 expression in cardiac rejection. Transplantation 1996; 61: 1610-1617.

47 Keogh A, Richardson M, Ruygrok P, Spratt P, Galbraith A, O'Driscoll G et al. Sirolimus in de novo heart transplant recipients reduces acute rejection and prevents coronary artery disease at 2 years: a randomized clinical trial. Circulation 2004; 110: 2694-2700.

48 Lindenfeld J, Miller GG, Shakar SF, Zolty R, Lowes BD, Wolfel EE et al. Drug therapy in the heart transplant recipient: part I: cardiac rejection and immunosuppressive drugs. Circulation 2004; 110: 3734-3740.

49 Khalil A, Carrier M, Latour JG, Pelletier LC. Cyclosporin A-induced coronary artery vasoconstriction through myogenic and endotheliumdependent mechanisms. Circulation 1996; 94: II308-II311.

50 Mazanet MM, Neote K, Hughes CC. Expression of IFN-inducible T cell alpha chemoattractant by human endothelial cells is cyclosporin Aresistant and promotes $T$ cell adhesion: implications for cyclosporin A-resistant immune inflammation. J Immunol 2000; 164: 53835388.

51 Dogan A, MacDonald TT, Spencer J. Cyclosporin A enhances T cellmediated induction of E-selectin. Eur J Immunol 1993; 23: 2922-2926.

52 Jeanmart H, Malo O, Carrier M, Nickner C, Desjardins N \& Perrault LP et al. Comparative study of cyclosporine and tacrolimus vs newer immunosuppressants mycophenolate mofetil and rapamycin on coronary endothelial function. J Heart Lung Transplant 2002; 21: 990-998.

53 Weigel G, Bertalanffy P, Dubsky P, Griesmacher A, Wolner E. Mycophenolic acid influences T helper 2 (Th2) cytokine induced expression of intercellular cell adhesion molecule-1 (ICAM-1) on human endothelial cells. Clin Chem Lab Med 1999; 37: 253-257.

54 Hilger RA, Koller M, Konig W. Immunosuppressive agents enhance the cytokine-induced priming of inflammatory cells. Int Arch Allergy Immunol 1995; 107: 259-260.

55 Zoncu R, Efeyan A \& Sabatini DM. mTOR: from growth signal integration to cancer, diabetes and ageing. Nat Rev Mol Cell Biol $2011 ; 12: 21-35$.

56 Cho D, Signoretti S, Regan M, Mier JW \& Atkins MB. The role of mammalian target of rapamycin inhibitors in the treatment of advanced renal cancer. Clin Cancer Res 2007; 13: 758s-763s.

57 Albiges L, Chamming's F, Duclos B, Stern M, Motzer RJ, Ravaud A et al. Incidence and management of mTOR inhibitor-associated pneumonitis in patients with metastatic renal cell carcinoma. Annals of Oncology 2012; 23: 1943-1953.

58 Martinet W, Verheye S, De Meyer I, Timmermans JP, Schrijvers DM, Van Brussel I et al. Everolimus triggers cytokine release by macrophages: rationale for stents eluting everolimus and a glucocorticoid. Arterioscler Thromb Vasc Biol 2012; 32: 1228-1235.

59 Haddad LE \& Sirois MG. Angiopoietin-1 upregulates de novo expression of IL-1beta and il1-ra, and the exclusive release of il1-ra from human neutrophils. PLoS One 2014; 9: e88980.

60 Gomez-Cambronero J. Rapamycin inhibits GM-CSF-induced neutrophil migration. FEBS Lett 2003; 550: 94-100.

61 Sasakawa Y, Sakuma S, Higashi Y, Sasakawa T, Amaya T \& Goto T et al. FK506 suppresses neutrophil chemoattractant production by peripheral blood mononuclear cells. Eur J Pharmacol 2000; 403: 281-288.

62 Lorne E, Zhao X, Zmijewski JW, Liu G, Park YJ, Tsuruta Y et al. Participation of mammalian target of rapamycin complex 1 in Tolllike receptor 2- and 4-induced neutrophil activation and acute lung injury. Am J Respir Cell Mol Biol 2009; 41: 237-245.

63 Baggiolini M, Dewald B, Moser B. Human chemokines: an update. Annu Rev Immunol 1997; 15: 675-705.

64 Freyer DR, Morganroth ML, Todd RF 3rd. Surface Mo1 (CD1 1b/CD18) glycoprotein is up-modulated by neutrophils recruited to sites of inflammation in vivo. Inflammation 1989; 13: 495-505.

65 Lo SK, Detmers PA, Levin SM, Wright SD. Transient adhesion of neutrophils to endothelium. J Exp Med 1989; 169: 1779-1793.

66 Sako D, Chang XJ, Barone KM, Vachino G, White HM, Shaw G et al. Expression cloning of a functional glycoprotein ligand for P-selectin. Cell 1993; 75: 1179-1186.

67 Hauser IA, Johnson DR, Thevenod F, Goppelt-Strube M. Effect of mycophenolic acid on TNF alpha-induced expression of cell adhesion molecules in human venous endothelial cells in vitro. $\mathrm{Br} \mathrm{J}$ Pharmacol 1997; 122: 1315-1322.

\section{(c) (1) (5) (2)}

This work is licensed under a Creative Commons Attribution-NonCommercial-ShareAlike 3.0 Unported License. The images or other third party material in this article are included in the article's Creative Commons license, unless indicated otherwise in the credit line; if the material is not included under the Creative Commons license, users will need to obtain permission from the license holder to reproduce the material. To view a copy of this license, visit http://creativecommons.org/licenses/by-nc-sa/3.0/ 